\title{
Postoperative Hemorrhage
}

National Cancer Institute

\section{Source}

National Cancer Institute. Postoperative Hemorrhage. NCI Thesaurus. Code C34938.

Bleeding occurring after completion of a surgical procedure, which may occur

immediately or may be delayed, and which may or may not be wound related. 